\title{
The Application of the Model of High-Speed Pixel Clustering in Problems of Preprocessing of the Images of the Remote Sensing of the Earth ${ }^{\star}$
}

\author{
Igor Khanykov ${ }^{[0000-0001-6284-0839]}$ \\ St. Petersburg Federal Research Center of the Russian Academy of Sciences \\ igkeiias.spb.su \\ http://www.machinelearning.ru/wiki/index.php?title=User:Khan
}

\begin{abstract}
The purpose of the research is to use the modified Ward's method in high-speed processing of full-HD images of the remote sensing of the Earth. The classical Ward's method is modified by dividing the computational process into three successive stages. The first stage quickly builds a coarse hierarchy of approximations. The second stage performs a quality improvement of the specified partition for a fixed number of colors (clusters). The third stage is the clustering of the superpixels using the Ward's method. The software-algorithmic toolkit consists of four operations on clusters of pixels and image segments: merge operation joins together two clusters; divide operation reversibly disjoins the selected cluster into two; split operation extracts the part of the cluster into individual cluster; correct operation reclassifies pixels by extracting from one cluster and inserting into another cluster. The quality is assessed by the total squared error. The quality improvement is provided by iterative execution of a combination of merge and divide operations of pixel clusters, in particular image segments. One of the clusters (segments) is divided in two and a pair of other mismatched with it is combined into one according to the criterion of the minimum increment of the total squared error. The proposed modified Ward's method is appropriate in processing of fullHD images of the remote sensing of the Earth. The results of processing in pure segmentation and clustering modes are compared. The proposed pixel clustering model is appropriate in high-speed processing of the full-HD images. The pixel clustering in comparison with image segmentation allows to define in more detail both the contours of objects of interest and their internal structure.
\end{abstract}

Keywords: Image Segmentation · Pixel Clustering $\cdot$ High-Speed Clustering $\cdot \mathrm{Su}-$ perpixels $\cdot$ Approximation Hierarchy $\cdot$ Earth Remote Sensing.

\section{Introduction}

The segmentation task refers to the stage of preliminary processing of the images. It consists in dividing the image into disjoint areas based on the uniformity of characteristics (brightness or color of pixels). The segmentation is applicable in many practical

\footnotetext{
Copyright (C) 2020 for this paper by its authors. Use permitted under Creative Commons License Attribution 4.0 International (CC BY 4.0).

* Publication is supported by RFBR grant 19-07-00844
} 


\section{I. Khanykov}

areas, including remote sensing of the Earth. One of the common approaches to segmentation of satellite images is based on the use of data clustering algorithms. The paper [1] presents the features of clustering satellite images, among which large amount of data, lack of a priori information about the number and probabilistic characteristics of classes, the presence of "noise" and emissions in the data are distinguished.

Image segmentation (pixel clustering in general) consists in dividing the original image into embedded images of "objects" for the purpose of further analysis of attributes and recognition. Filling each embedded image with the same pixels with an average brightness value converts the original image to its approximation. The quality of the partition and the corresponding approximation of an image of $N$ pixels is estimated by the standard deviation $\sigma$ of the approximation pixels from the image pixels or the total square error $E=3 N \sigma^{2}$, where coefficient 3 takes into account the number of color components in the image. At the segmentation, the pixels of each embedded image constitute a single connected segment. At clustering pixels, it is assumed that the embedded image may consist of several or many non-adjacent segments of the original image. The partition and approximation for a given number of pixel clusters, in particular image segments, is considered to be optimal if it corresponds to the minimum possible value of the total squared error $E$ or standard deviation $\sigma$. Then the objects are defined as clusters or segments of the optimal image approximation.

Present paper is devoted to the application of the modification [2,3] of one of the classical cluster analysis methods $[4,5]$ for preprocessing digital images of Earth remote sensing at the segmentation stage. Section 2 provides a brief overview of classical cluster analysis techniques as applied to image processing. It also compares the classical methods of cluster analysis applicable to the problem of digital image segmentation. Their advantages and disadvantages are given. The problem to overcome the computational complexity of the classical Ward's method is set. Section 3 describes a modification of the classical Ward's method (model of high-speed pixel clustering). A typical block scheme of the sequence of algorithms is disclosed. It overcomes the shortcomings of the classical Ward's method by means of intermediate processing. Section 4 presents the software-algorithmic toolkit of the model. The idea of reversible operations in image processing is described. Section 5 discusses the experimental results. The table shows the time spent on processing test images of several sizes in different modes. The final section summarizes the work done.

\section{Overview and comparison}

The Ward's [6], Otsu [7], K-means [8] methods and the Mumford-Shah segmentation model $[9,10]$ take special place among classical data clustering methods. These methods are well known. There are many modifications. For example, a new method for generating the center of a cluster by reducing the mean square error of the final cluster without significantly increasing the execution time of the K-means method is presented in [11]. The mechanism for finding the initial centroids, which ensures the efficient assignment of points to suitable clusters is improved in [12,13]. Clustering by the K-means method is combined with the fuzzy logic of C-means method in [14]. Fuzzy logic methods are combined with threshold processing methods in [15]. It also 
presents a classification of threshold image segmentation methods. The complexity of the Mumford-Shah model is overcome in [16] by replacing a piecewise smooth function with a piecewise constant function.

In [17], devoted to the selection of hyperspectral image characteristics for spatial and spectral clustering by the fuzzy C-means logic method, the Ward's method is used as an agglomerative algorithm for constructing a hierarchy in which each spectral band is considered as a cluster. The iterative merging process is repeated until the desired number of clusters is reached. In [18], clustering methods are used to identify homogeneous areas of watersheds in remote sensing problems. The Ward's method, which demonstrated excellent quality results, was used to form a two-dimensional map of objects. In [19], an algorithm for recognizing the regions of lunar seas based on the Ward's method is proposed.

Despite the fact that the Ward's method returns results of acceptable quality by the total squared error, it is limited in used in image processing due to the high computational complexity.

The following challenging requirements are applied for the modern algorithms of image segmentation [20]:

1) The lack of a priori information about the objects of interest.

2) The presence of the established quality criterion allowing to evaluate the obtained image partition into clusters/segments.

3) The possibility of segmenting the image into each number of colors/clusters from 1 to $N$, where $N$ is the total number of pixels in the considered image.

4) The real-time processing.

5) The adequacy of the results, consisting in correspondence of the selected segments/clusters to the boundaries and areas of objects in the image.

Most of the above requirements are met by a group of cluster methods. The most common are Ward's method [6], Otsu [7], K-means [8] and the Mumford-Shah segmentation model $[9,10]$. The mentioned methods have a number of common features. None of the listed methods requires a priori information about the image. These methods are suitable for image processing of any content and subject matter. The above methods minimize the established quality functional. Its value serves as an indicator of the quality of splitting the image into pixel clusters, image segments.

The difference of the considered cluster methods reveals in the way the image is divided into clusters of pixels or segments. The K-means method divides image pixels into an earlier predetermined and fixed number of clusters. The Otsu method, the Mumford-Shah segmentation model and the Ward's method generate a set of partitions of the original image from 1 to $N$, where $N$ is the total number of pixels in the original image. In contrast to Ward's method that considers all the combinations of pixel clusters, the Mumford-Shah segmentation model considers only pairs of adjacent segments of the image.

The compared methods have different computational complexity and, consequently, possess different possibility of processing images in real time. If the classical Otsu method takes linear time to split pixels into two clusters, then in the general case its computational complexity grows exponentially with an increase of considered number of thresholds. The time taken by the K-means method for the division an image into 


\section{I. Khanykov}

a fixed number of clusters depends quadratically on the preset value of the number of clusters. The Mumford-Shah model segments the image in nearly real time. The computational complexity of the classical Ward's method increases quadratically with the rise of the number of the considered clusters of pixels that makes it difficult to apply the method directly to the image processing.

The Ward's method and the Mumford-Shah segmentation model support a hierarchical data structure. It stores information about the sequence of merges of pixel clusters or image segments, the values of total square errors, the values of the number of pixels and the average intensities for each newly formed pixel cluster or image segment. The hierarchical data structure allows both to perform a "rollback" operation into the past state, as well as to find a new partition characterized by a smaller value of the total squared error.

The classical Ward's method satisfies most of listed requirements applicable to challenging image segmentation algorithms. But the high computational complexity significantly limits its application in image processing. The considered in further section modification of the classical Ward's method allows to process images in image segmentation and pixel clustering tasks by means of dividing whole process into three successive stages.

\section{The modification of the classical Ward's method}

Among the methods of cluster analysis applicable in image processing, the classical Ward's method takes special place. It processes color images and returns adequate results, but the high computational complexity characteristic of the method significantly limits its application. Shown in Fig. 1, the scheme for the high-speed clustering of image pixels overcomes this drawback by dividing the processing process into three typical stages.

The first stage "a" quickly builds a rough hierarchy of connected segments, the generation of which is available in two different variants. The first variant is to use the Mumford-Shah model $[9,10]$ for enlarging segments at each step. The second variant is to divide the image into fragments by a regular grid for processing them as independent images by the classical Ward's method with the subsequent merging of hierarchies into one [21].

The second stage "b" forms $N$ sp superpixels, in fact, performing an improvement in the quality of a given partition with a fixed number of colors (clusters). For this task, two basic algorithms have been developed: $S I$-method (Segmentation Improvement) [2] and $K$-meanless method (K-means-without-means method) [3]. Many software implementations are due to both the possibility of a combination of a pair of basic $S I$ and $K$-meanless methods (separately, sequentially, cyclically), and versions of the methods themselves (segmental, cluster).

At the third stage "c", superpixels are clustered by the Ward's method [6]. 


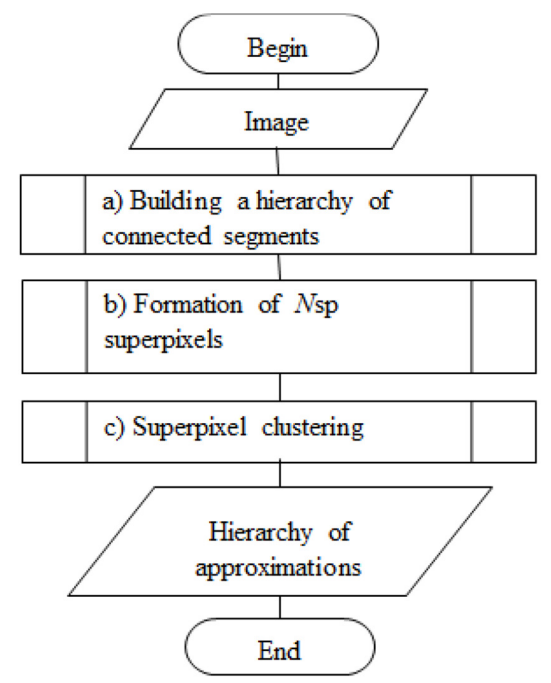

Fig. 1. Three-stage flowchart of the high-speed clustering of the image pixels.

\section{The software-algorithmic toolkit}

The basis of the software and algorithmic toolkit for the high-speed pixel clustering scheme [22] is four operations with pixel clusters, in particular, image segments, which are used to minimize the total squared error $E$ or standard deviation $\sigma$ :

- the "merge" operation of merging two clusters

$$
\Delta E_{\text {merge }}=\frac{n_{1} n_{2}}{n_{1}+n_{2}}\left\|I_{1}-I_{2}\right\|^{2} .
$$

The criterion for operation (1) execution:

$$
\Delta E_{\text {merge }}=\min .
$$

- the "divide" operation to split a cluster in two

$$
\Delta E_{\text {divide }}(1)=-\Delta E_{\text {merge }}\left(1^{\prime}, 1^{\prime \prime}\right) \text {, }
$$

where $1=1^{\prime} \bigcup 1^{\prime \prime}$.

- the "split" operation of selection of any subset of $k<n_{1}$ pixels from $n_{1}$ pixels of cluster 1 into a separate cluster

$$
\Delta E_{\text {split }}=-\frac{k n_{1}}{n_{1}-k}\left\|I-I_{1}\right\|^{2} \leq 0,
$$

where $I_{1}$ and $I$ are the three-component average brightnesses of the discussed $n_{1}$ and $k$ pixels. 
6 I. Khanykov

- "correct" operation of reclassification of pixels by extraction them from one cluster and assigning them to another cluster

$$
\Delta E_{\text {correct }}=\frac{k n_{2}}{n_{2}+k}\left\|I-I_{2}\right\|^{2}-\frac{k n_{1}}{n_{1}-k}\left\|I-I_{1}\right\|^{2}
$$

where $I$ is the average value of reclassified $k$ pixels, and $I_{1}, I_{2}$ are the average values of pixels of clusters 1 and 2. Criteria for the (5) operation is:

$$
\Delta E_{\text {correct }}=\min <0 .
$$

The first two operations (1), (3) are used to build the hierarchy. A pair of other operations (4), (5) is used in hierarchy transformations.

The combination of "merge\&divide" operations (1), (3) improves image quality in the $S I$-method by dividing one of the segments (numbered 1) and merging the other two mismatched (numbered 2 and 3). SI-method is executed by the criterion [2]:

$$
\Delta E_{\text {divide }}(1)=\Delta E_{\text {merge }}(2,3)=\min <0 .
$$

At each iteration, a triple of segments is selected that provide the maximum drop in the total squared error, and the process of combined merging/dividion of the adjacent segments continues until three segments are found that satisfy the condition. Otherwise, the processing ends. For applications of the $S I$-method, it is important that the number of segments in the resulting approximation coincides with the number of segments in the original approximation.

Using the above operations (1), (3), (4), (5) and their combinations [22], a binary hierarchy of clusters or segments is built, and a hierarchical sequence of quasi-optimal image partitions is formed into a sequential number of clusters from 1 to $N$. In this case, the hierarchy of clusters (segments) is considered to be given if for each cluster of at least one pixel, a pair of clusters is established into which this cluster is divided.

\section{Experimental results}

The application of the high-speed pixel clustering scheme is demonstrated by processing Earth remote sensing images taken from the Signal and Image Processing Institute of the University of Southern California (USC SIPI) database. In Fig. 2 the original image of the planet Earth is shown.

Series of Fig. 3-6 and Fig. 7-10 illustrate pixel clustering and image segmentation. The number of clusters/segments into which the original image is divided, as well as the value of the total squared error characterizing the quality of this partition is noted under each figure. The smaller value of total squared error, the better the quality of the partition. Note that pixel clustering gives a significantly more drop in the value of approximation error than the segmentation procedure. This can be noticed by comparing each subsequent partition of the image.

Fig. 11 shows grayscale aerospace image of the Pentagon. In the series of Fig. $12-$ 15 the details of the building appear more clearly with each subsequent splitting, than in a similar series of segmentations of Fig. $16-19$. 
The Application of the Model of High-Speed Pixel Clustering... 7

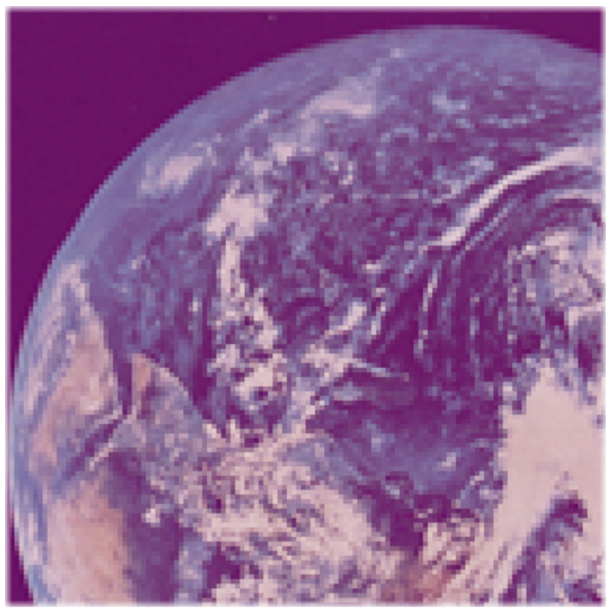

Fig. 2. The original image 2.1.11 "Earth from space" size of 512x512 pixels.

Pixel clustering, $N \mathrm{sp}=1000$

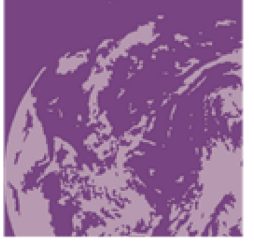

Fig. 3. $N \mathrm{cl}=2, \sigma=24,14763$

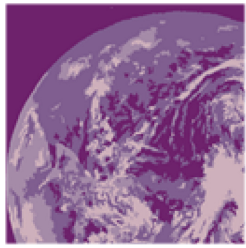

Fig. 5. $N \mathrm{cl}=4, \sigma=14,42725$

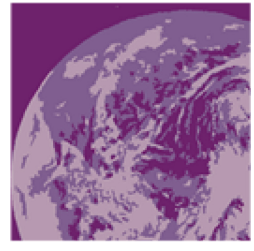

Fig. 4. $N \mathrm{cl}=3, \sigma=17,15115$

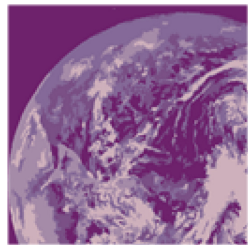

Fig. 6. $N \mathrm{cl}=5, \sigma=13,13059$

Image segmentation, $N \mathrm{sp}=1$

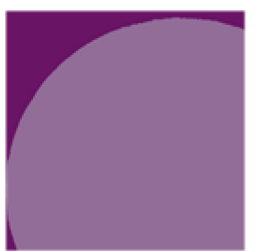

Fig. 7. $N \mathrm{sg}=2, \sigma=32,58404$

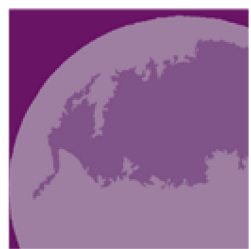

Fig. 8. $N s g=3, \sigma=29,19237$ 


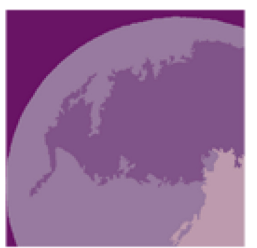

Fig. 9. $N$ sg $=4, \sigma=28,17634$

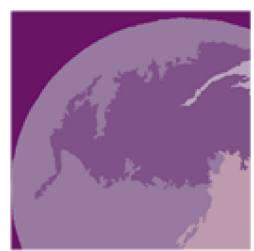

Fig. 10. $N$ sg $=5, \sigma=27,40356$

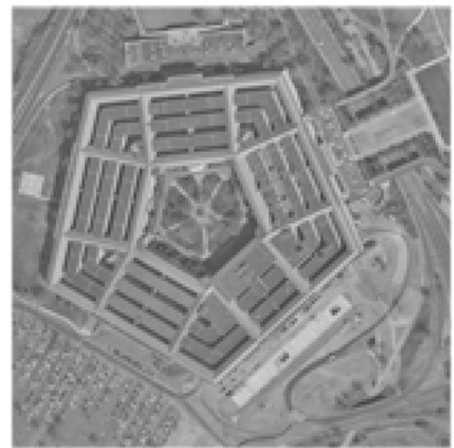

Fig. 11. The original image 3.2.25 "Pentagon" size of 1024x1024 pixels.

Pixel clustering, $N \mathrm{sp}=1000$

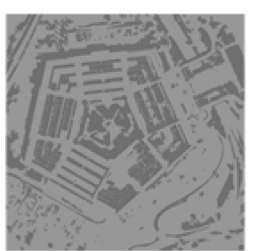

Fig. 12. $N \mathrm{cl}=2, \sigma=20,94362$

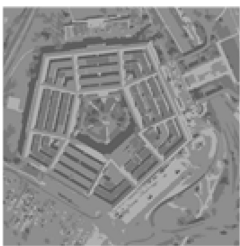

Fig. 14. $N \mathrm{cl}=4, \sigma=15,50541$

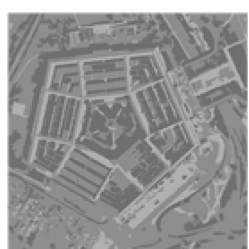

Fig. 13. $N \mathrm{cl}=3, \sigma=17,12895$

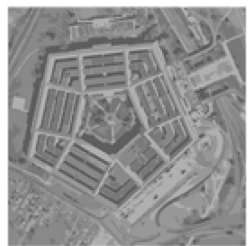

Fig. 15. $N \mathrm{cl}=5, \sigma=14,59845$ 


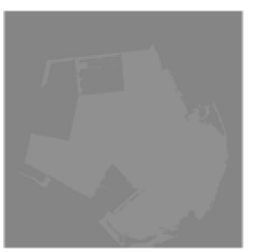

Fig. 16. $N \operatorname{sg}=2, \sigma=27,29347$

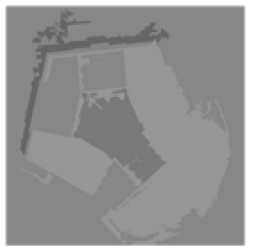

Fig. 18. $N \mathrm{sg}=4, \sigma=25,71989$

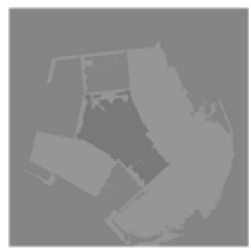

Fig. 17. $N$ sg $=3, \sigma=26,43707$

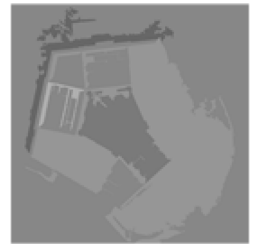

Fig. 19. $N \mathrm{sg}=5, \sigma=25,47795$

Fig. 20 depicts Washington in the infrared spectrum. The series of Fig. $21-24$ and Fig. $25-28$ show the results of pixel clustering and image segmentation. The details of the underlying surface and the outlines of the river are visible already on the second clustered partition (Fig. 21).

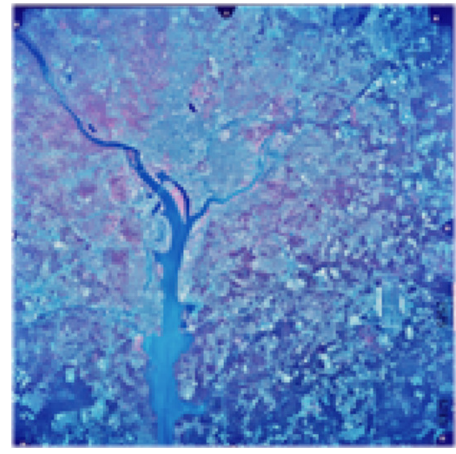

Fig. 20. The original image wash-ir "Washington D.C. (infra-red)" size of 2250x2250 pixels.

The Table 1 shows the time costs for processing the images presented in the work in various modes: pixel clustering and image segmentation. The first column shows the name of the standard test image from the open USC-SIPI database. The second column indicates the size of the image sides in pixels. All images are square. The third column sets the Nsp number of superpixels - the detail parameter, which takes values in the 
Pixel clustering, $N \mathrm{sp}=1000$

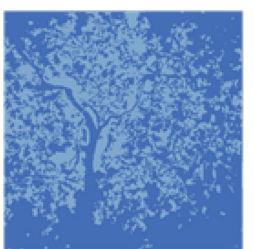

Fig. 21. $N \mathrm{cl}=2, \sigma=36,29479$

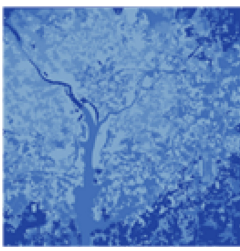

Fig. 23. $N \mathrm{cl}=4, \sigma=31,51019$

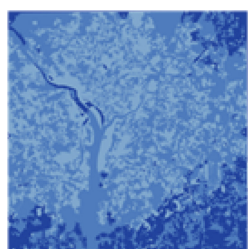

Fig. 22. $N \mathrm{cl}=3, \sigma=32,73039$

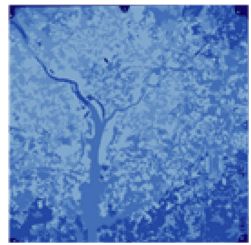

Fig. 24. $N \mathrm{cl}=5, \sigma=30,90131$

Image segmentation, $N \mathrm{sp}=1$

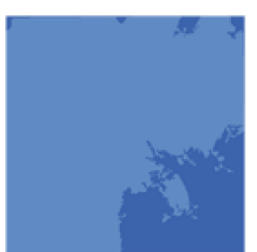

Fig. 25. $N \mathrm{sg}=2, \sigma=41,12131$

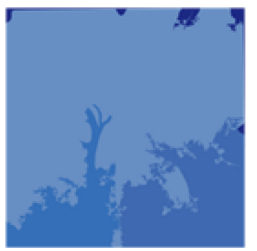

Fig. 27. $N$ sg $=4, \sigma=39,00807$

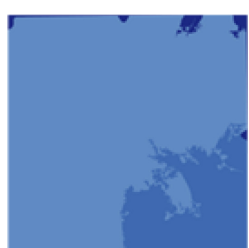

Fig. 26. $N$ sg $=3, \sigma=40,51840$

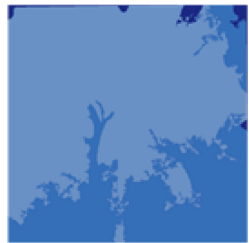

Fig. 28. $N \operatorname{sg}=5, \sigma=38,56550$ 
range from 1 to $N$ the number of pixels in the image in total. When $N$ sp $=1$, the program operates in pure segmentation mode, considering only pairs of adjacent pixels as in the modified Mumford-Shah model [9, 10,22]. Similarly, for $N \mathrm{sp}=N$, the program operates in the pure clustering mode with quadratic computational complexity as in the classical Ward's method [6]. Practice shows that a compromise value of $N \mathrm{sp}=1000$ is enough to highlight the details of an object without losing time on image processing. The fourth, fifth, and sixth columns show the time costs and image processing speeds at each stage of the algorithm. The last seventh column shows the total time of the algorithm for processing the image in a given mode.

Table 1. Comparison of time costs in pixel clustering and image segmentation modes.

\begin{tabular}{|c|c|c|c|c|c|c|}
\hline Image & Dimensions & $N \mathrm{sp}$ & $\begin{array}{l}\text { Stage "a" } \\
{[t, \text { sec; }} \\
v, \text { pix/sec }]\end{array}$ & $\begin{array}{l}\text { Stage "b" } \\
{[t, \text { sec; }} \\
v, \text { pix/sec }]\end{array}$ & $\begin{array}{l}\text { Stage "c" } \\
{[t, \text { sec; }} \\
v, \text { pix/sec }]\end{array}$ & $\begin{array}{l}\text { Time, } \\
\text { sec }\end{array}$ \\
\hline $\begin{array}{l}2.1 .11 \text { "Earth } \\
\text { from space" }\end{array}$ & $\begin{array}{l}512 \mathrm{x} \\
512\end{array}$ & 1 & $\begin{array}{l}8,790 \\
29823\end{array}$ & & & 8,790 \\
\hline $\begin{array}{l}2.1 .11 \text { "Earth } \\
\text { from space" }\end{array}$ & $\begin{array}{l}512 x \\
512\end{array}$ & 1000 & $\begin{array}{l}9,046 \\
28979\end{array}$ & $\begin{array}{l}0,023 ; \\
11397565\end{array}$ & $\begin{array}{l}1,305 ; \\
200877\end{array}$ & 10,374 \\
\hline 3.2.25 Pentagon & $\begin{array}{l}1024 x \\
1024\end{array}$ & 1 & $\begin{array}{l}60,627 \\
17296\end{array}$ & & & 60,627 \\
\hline 3.2.25 Pentagon & $\begin{array}{l}1024 x \\
1024\end{array}$ & 1000 & \begin{tabular}{|l|}
59,$832 ;$ \\
17525
\end{tabular} & $\begin{array}{l}0,041 ; \\
25575024\end{array}$ & $\begin{array}{l}1,61 ; \\
650885\end{array}$ & 61,484 \\
\hline $\begin{array}{l}\text { wash-ir Washington } \\
\text { D.C. (infra-red) }\end{array}$ & $\begin{array}{l}2250 x \\
2250\end{array}$ & 1 & $\begin{array}{l}202,862 \\
24955\end{array}$ & & & 202,862 \\
\hline $\begin{array}{l}\text { wash-ir Washington } \\
\text { D.C. (infra-red) }\end{array}$ & $\begin{array}{l}2250 x \\
2250\end{array}$ & 1000 & $\begin{array}{l}206,872 \\
24472\end{array}$ & $\begin{array}{l}0,061 \\
82991803\end{array}$ & $\begin{array}{l}0,909 ; \\
5569307\end{array}$ & 207,842 \\
\hline
\end{tabular}

\section{Conclusion}

The paper is devoted to the application of the modified Ward's method in the processing of Earth remote sensing images. The overview of the application of classical methods of cluster analysis in image processing is provided. The actual requirements for the challenging algorithms of image segmentation (pixel clustering) are provided. The comparison of classical methods of data clustering according to the given requirements is carried out. The relevance of the modification of the classical Ward's method is justified. The block-scheme of the computational process that overcomes the computational complexity of the classical Ward's method is presented. The software-algorithmic toolkit is given and the options for implementing the blocks of the model are described. The capabilities of high-speed pixel clustering are demonstrated by the examples of processing series of images of the remote sensing of the Earth. 


\section{I. Khanykov}

The peculiarity of the modified Ward's method is the bypass of the computational complexity by dividing the process into three sequential stages. It allows to implement the idea of Ward's method in pixel clustering and image segmentation tasks.

It was established, that pixel clustering in comparison with image segmentation makes it possible to define both the contours of objects of interest and their internal structure in more detail. The time spent on image processing in the clustering mode with a compromise value of the input parameter of the number of superpixels $N \mathrm{sp}=1000$ is close to the time required for image processing in the pure segmentation mode with $N \mathrm{sp}=1$.

The statistical data of image processing is summarized in Table 1.

\section{References}

1. Pestunov I.A., Sinyavskiy Yu.N. [Clustering Algorithms in Satellite Images Segmentation Tasks]. Bulletin of Kemerovo State University. 4(52), pp. 110-125 (2012). (in Russ.) https://cyberleninka.ru/article/v/algoritmy-klasterizatsii-v-zadachah-segmentatsiisputnikovyh-izobrazheniy. Last accessed 15 Aug 2020

2. Kharinov, M.V., Khanykov, I.G.: [Optimization of Piecewise Constant Approximation for Segmented Image]. SPIIRAS Proceedings. 3(40), pp. 183-202 (2015) (in Russ.) https://doi.org/10.15622/sp.40.12

3. Khanykov, I.G., Kharinov, M.V., Patel, C.: Image segmentation improvement by reversible segment merging. International Conference on Soft Computing and its Engineering Applications (icSoftComp), IEEE, pp. 1-8 (2017). https://doi.org/10.1109/ICSOFTCOMP.2017.8280096

4. Aivazyan, S.A., Bukhshtaber, V.M., Enyukov, I.S., Meshalkin, L.D.: [Applied statistics: Classification and reduction of dimension]. Moskva: Finansy i statistika. 607 p. (1989) (in Russ.)

5. Mandel', I.D.: [Cluster Analysis] Moskva: Finansy i statistika. 176 p. (1988) (in Russ.)

6. Ward, J.H.Jr.: Hierarchical grouping to optimize an objective function. J. Am. Stat. Assoc. 58(301), 236-244 (1963). https://doi.org/10.1080/01621459.1963.10500845

7. Otsu, N.A.: Threshold selection method from gray-level histograms. IEEE Transactions on Systems, Man and Cybernetics 9(1), 62-66 (1979). https://doi.org/10.1109/TSMC.1979.4310076

8. Lloyd, S.P.: Least squares quantization in PCM. IEEE Transactions on Information Theory 28(2), pp. 129-137 (1957/1982). https://doi.org/10.1109/TIT.1982.1056489

9. Mumford, D., Shah, J.: Boundary detection by minimizing functionals. IEEE Conference on Computer Vision and Pattern Recognition, vol. 17, pp. 137-154 (1985).

10. Mumford, D., Shah, J.: Optimal approximations by piecewise-smooth functions and associated variational problems. Communications on pure and applied mathematics 42(5), pp. 577-685 (1989). https://doi.org/10.1002/cpa.3160420503

11. Purohit, P., Joshi, R.A.: New Efficient Approach towards k-means Clustering Algorithm. In International Journal of Computer Applications. 65(11), pp. 125-129 (2013). https://pdfs.semanticscholar.org/99bb/dc0435b10476f61a778e0ab00301704c647c.pdf. Last accessed 19 May 2020

12. Yedla, M., Pathakota, S.R., Srinivasa, T.M.: Enhanced K-means Clustering Algorithm with Improved Initial Center. In International Journal of Science and Information Technologies. 1(2), pp. 121-125 (2010)

13. Nazeer, K.A.A., Sebastian, M.P.: Improving the Accuracy and Efficiency of the k-means Clustering Algorithm. In Proceedings of the World Congress on Engineering (2009) 
14. Jose, A, Ravi, S, Sambath, M.: Brain tumor segmentation using k-means clustering and fuzzy c-means algorithms and its area calculation. International Journal of Innovative Research in Computer and Communication Engineering 2(3), pp. 3496-3501 (2014)

15. Das, A., Sabut, S.K.: Kernelized fuzzy C-means clustering with adaptive thresholding for segmenting liver tumors. Proceedings on Computer Science, vol. 92, pp. 389-395 (2016). https://doi.org/10.1016/j.procs.2016.07.395

16. Chan, T.F., Vese, L.A.: Active contours without edges. IEEE Transactions on Image processing, 10(2), pp. 266-277 (2001). http://w3.mi.parisdescartes.fr/ lomn/Cours/CV/SeqVideo/Articles/ChanLevelSet.pdf

17. Salem, M.B., Ettabaa, K.S., Bouhlel M.S.: Hyperspectral image feature selection for the fuzzy c-means spatial and spectral clustering. In International Image Processing, Applications and Systems (IPAS) IEEE, pp. 1-5 (2016). https://doi.org/10.1109/ipas.2016.7880114

18. Sardooi, E.R., Azareh, A., Choubin, B., Barkhori, S., Singh, V.P., Shamshirband, S. Applying the remotely sensed data to identify homogeneous regions of watersheds using a pixel-based classification approach. Applied Geography, vol. 111 (2019). https://doi.org/10.1016/j.apgeog.2019.102071

19. Xie, T, Jiang, H, Wang, J, Tian, X., Xu, A.A.: A new recognition algorithm of the lunar mare area basing on the DEM contrast. In International Conference on Advanced Materials and Engineering Structural Technology, pp. 1-4 (2015 Apr 25)

20. Porshnev S.V., Levashkina A.O. [Universal Classification of Image Segmentation Algorithms]. Journal of scientific publications of post-graduate and doctorate students. vol. 3, pp. 163-172 (2008). (in Russ.)

21. Kharinov, M.V., Khanykov, I.G.: [Utilization of Ward's Method for Clustering of Pixels of Color Image]. BSU bulletin. Mathematics, Informatics, vol. 4, pp. 34-42 (2016). (in Russ.) https://doi.org/10.18101/2304-5728-2016-4-34-42.

22. Kharinov, M.V.: [A generalization of three approaches to an optimal segmentation of digital image]. Trudy SPIIRAN, vol. 25, pp. 294-316. (2013). (in Russ.). http://www.mathnet.ru/links/d13b2a4e766e53f82eb216ba6367bf29/trspy552.pdf. Last accessed 15 Aug 2020 